\title{
PHILONIS ALEXANDRINI
}

\section{OPERA QVAE SVPERSVNT}

\author{
VOL. V
}

EDIDIT

LEOPOLDVS COHN

ADIECTAE SUNT TABULAE PHOTOTYPICAE DUAE

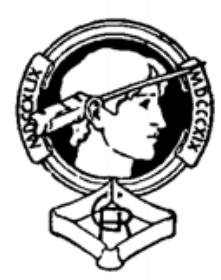

BEROLINI

TYPIS ET IMPENSIS GEORGII REIMERI 


\section{PHILONIS ALEXANDRINI}

\section{OPERA QVAE SVPERSVNT}

EDIDERVNT

LEOPOLDVS COHN ET PAVLVS WENDLAND

$$
\text { VOL. V }
$$

CONTINENS DE SPECIALIBVS LEGIBVS LIB. I-IV

DE VIRTVTIBTS

DE FORTITVDINE

DE HVMANITATE

DE PAENITENTIA

DE NOBILITATE

DE PRAEMIIS ET POENIS

DE EXSECRATIONIBVS

BEROLINI

TYPIS ET IMPENSIS GEORGII REIMERI 


\title{
PHILONIS ALEXANDRINI
}

\section{OPERA QVAE SVPERSVNT}

\author{
VOL. V
}

EIDIDIT

LEOPOLDVS COHN

ADIECTAE SVNT TABVLAF PHOTOTYPICAE DVAE

\section{BEROLINI}

TYPIS ET IMPANSIS GEORGII REIMERI 


\section{PHILONIS OPERA}

